PROCEEDINGS OF THE

AMERICAN MATHEMATICAL SOCIETY

Volume 128, Number 2, Pages 407-411

$\mathrm{S}$ 0002-9939(99)05020-0

Article electronically published on July 6, 1999

\title{
ASYMPTOTIC BEHAVIOUR OF CASTELNUOVO-MUMFORD REGULARITY
}

\author{
VIJAY KODIYALAM
}

(Communicated by Wolmer V. Vasconcelos)

\begin{abstract}
Let $S$ be a polynomial ring over a field. For a graded $S$-module generated in degree at most $P$, the Castelnuovo-Mumford regularity of each of (i) its $n^{\text {th }}$ symmetric power, (ii) its $n^{\text {th }}$ torsion-free symmetric power and (iii) the integral closure of its $n^{\text {th }}$ torsion-free symmetric power is bounded above by a linear function in $n$ with leading coefficient at most $P$. For a graded ideal $I$ of $S$, the regularity of $I^{n}$ is given by a linear function of $n$ for all sufficiently large $n$. The leading coefficient of this function is identified.
\end{abstract}

Let $S=k\left[x_{1}, \cdots, x_{d}\right]$ be a polynomial ring over a field $k$ with its usual grading, i.e., each $x_{i}$ has degree 1 , and let $\mathfrak{m}$ denote the maximal graded ideal of $S$. Let $N$ be a finitely generated non-zero graded $S$-module. The Castelnuovo-Mumford regularity of $N$, denoted $\operatorname{reg}(N)$, is defined to be the least integer $m$ so that, for every $j$, the $j^{\text {th }}$ syzygy of $N$ is generated in degrees $\leq m+j$. By Hilbert's syzygy theorem, $N$ has a graded free resolution over $S$ of the form

$$
0 \rightarrow F_{k} \rightarrow \cdots \rightarrow F_{1} \rightarrow F_{0} \rightarrow N \rightarrow 0
$$

where $F_{i}=\bigoplus_{j=1}^{t_{i}} S\left(-a_{i j}\right)$ for some integers $a_{i j}$ — which we will refer to as the twists of $F_{i}$. Then, $\operatorname{reg}(N) \leq \max _{i, j}\left\{a_{i j}-i\right\}$ with equality holding if the resolution is minimal. For other equivalent definitions and properties of this invariant, see [Snb].

For a graded ideal $I$ in $S$, the behaviour of the regularity of $I^{n}$ as a function of $n$ has been of some interest. If $I$ defines a smooth complex projective variety, it is shown in [BrtEinLzr, Proposition 1] using the Kawamata-Viehweg vanishing theorem that $\operatorname{reg}\left(I^{n}\right) \leq P n+Q$ where $P$ is the maximal degree of a minimal generator of $I$ and $Q$ is a constant expressed in terms of the degrees of generators of $I$. In [GrmGmgPtt, Theorem 1.1] and in [Chn, Theorem 1] it is shown that if $\operatorname{dim}(R / I) \leq 1$, then $\operatorname{reg}\left(I^{n}\right) \leq n \cdot \operatorname{reg}(I)$ for all $n \in \mathbb{N}$. In [Chn, Conjecture 1], this is conjectured to be true for an arbitrary graded ideal. Supporting this conjecture is the result of [Swn, Theorem 3.6] that $\operatorname{reg}\left(I^{n}\right) \leq P n$ for some constant $P$ and for all $n \in \mathbb{N}$. The method of proof makes it difficult to explicitly identify such a constant. For monomial ideals, such a $P$ is explicitly calculated in [SmtSwn, Theorem 3.1] and improved upon in [HoaTrn, Corollary 3.2].

We show that with $S$ and $N$ as above, the regularity of $\operatorname{Sym}_{n}(N)$ - and related modules - is bounded above by a linear function of $n$ with leading coefficient at

Received by the editors October 28, 1997 and, in revised form, April 15, 1998.

1991 Mathematics Subject Classification. Primary 13D02; Secondary 13D40.

(C)1999 American Mathematical Society 
most the maximal degree of a minimal generator of $N$. For a graded ideal $I$ of $S$, we get the sharper result that $\operatorname{reg}\left(I^{n}\right)$ is actually given by a linear function of $n$ for all sufficiently large $n$. The leading coefficient of this function is identified as a certain invariant $\rho(I)$ of $I$. This verifies Chandler's conjecture in the case $\operatorname{reg}(I)>\rho(I)$ and for all $n$ sufficiently large.

One of the main ingredients of the proof is an analysis of a bigrading $\left(=\mathbb{N}^{2}\right.$ grading) on the Rees ring, $S[I t]$, of a graded ideal $I \subseteq S$. This is defined by decreeing an element of $S[I t]$ to be homogeneous of bidegree $(p, n)$ if it is of the form $f t^{n}$ where $f$ is a homogeneous element of degree $p$ in $S$. For a recent application of this bigrading, see [CncHrzTrnVll].

Suppose that $I$ is generated minimally by homogeneous elements $f_{1}, \cdots, f_{k}$ in $S$ of degrees $p_{1}, \cdots, p_{k}$ respectively. Let $R=k\left[X_{1}, \cdots, X_{d}, T_{1}, \cdots, T_{k}\right]$ with bigrading defined by $\operatorname{deg}\left(X_{i}\right)=(1,0)$ and $\operatorname{deg}\left(T_{j}\right)=\left(p_{j}, 1\right)$. The natural map $R \rightarrow S[I t]$ defined by $X_{i} \mapsto x_{i}$ and $T_{j} \mapsto f_{j} t$ is then a surjective homomorphism of bigraded rings. In particular, $S[I t]$ is a cyclic bigraded $R$-module.

For a bigraded $R$-module $M=\bigoplus_{p, n \in \mathbb{N}} M_{(p, n)}$, define $M^{(n)}$ to be the graded $S$-module $\bigoplus_{p \in \mathbb{N}} M_{(p, n)}$ where $x_{i}$ acts as $X_{i}$ with its obvious grading. Note that $S[I t]^{(n)} \cong I^{n}$. The assignment $M \mapsto M^{(n)}$ is an exact functor. For $a, b \in \mathbb{N}$, define the twisted module $M(-a,-b)$ by $M(-a,-b)_{(p, n)}=M_{(p-a, n-b)}$. The crucial observation used in the proof is that

$$
R(-a,-b)^{(n)} \cong R^{(n-b)}(-a) \cong \bigoplus_{l_{1}+\cdots+l_{k}=n-b} S\left(-l_{1} p_{1}-\cdots-l_{k} p_{k}-a\right)
$$

as graded $S$-modules.

Theorem 1. Let $k$ be a field and $S=k\left[x_{1}, \cdots, x_{d}\right]$ graded as usual. Let $R=$ $k\left[X_{1}, \cdots, X_{d}, T_{1}, \cdots, T_{k}\right]$ with bigrading defined by $\operatorname{deg}\left(X_{i}\right)=(1,0)$ and $\operatorname{deg}\left(T_{j}\right)=$ $\left(p_{j}, 1\right)$ for some $p_{j} \in \mathbb{N}$. For a finitely generated bigraded $R$-module $M$, there exists a constant $Q$ so that reg $\left(M^{(n)}\right) \leq P n+Q$ for all $n \geq 1$ where $P=\max \left\{p_{1}, \cdots, p_{k}\right\}$.

Proof. By a bigraded version of Hilbert's syzygy theorem, the $R$-module $M$ has a bigraded free resolution of the form

$$
0 \rightarrow F_{k} \rightarrow \cdots \rightarrow F_{1} \rightarrow F_{0} \rightarrow M \rightarrow 0
$$

where $F_{i}=\bigoplus_{j=1}^{t_{i}} R\left(-a_{i j},-b_{i j}\right)$.

Applying the functor $(\cdot)^{(n)}$ to this resolution yields a graded free $S$-resolution of $M^{(n)}$ from which an upper bound on its regularity can be read off. Since

$$
F_{i}^{(n)} \cong \bigoplus_{j=1}^{t_{i}} \bigoplus_{l_{1}+\cdots+l_{k}=n-b_{i j}} S\left(-l_{1} p_{1}-\cdots-l_{k} p_{k}-a_{i j}\right),
$$

the maximal twist in $F_{i}$ is $\max _{j}\left\{P\left(n-b_{i j}\right)+a_{i j}\right\}$ where $P=\max \left\{p_{1}, \cdots, p_{k}\right\}$. Hence $\operatorname{reg}\left(M^{(n)}\right) \leq P n+Q$ with $Q=\max _{i, j}\left\{a_{i j}-P b_{i j}-i\right\}$.

As a matter of notation, for a graded $S$-module $N$, by $\theta(N)$ we will denote the maximal degree of a minimal generator of $N$. Equivalently, $\theta(N)=\operatorname{reg}(N / \mathfrak{m} N)$. For the definition and properties of integral closures of modules, see [Res].

Corollary 2. Let $S=k\left[x_{1}, \cdots, x_{d}\right]$ and $N$ be a finitely generated graded $S$-module with $\theta(N)=P$. Let $F_{n}(N)$ denote any one of:

(1) $\operatorname{Sym}_{n}(N)$ - the nth symmetric power of $N$. 
(2) $\mathrm{S}_{n}(N)$ - the nth symmetric power of $N$ modulo $S$-torsion.

(3) $\overline{\left(\mathrm{S}_{n}(N)\right)}$ - the integral closure of the module $\mathrm{S}_{n}(N)$.

Then, there exists $Q$ so that reg $\left(F_{n}(N)\right) \leq P n+Q$ for all $n \in \mathbb{N}$.

Proof. Let $N$ be generated by minimal generators in degrees $p_{1} \leq \cdots \leq p_{k}=P$ and let $R=k\left[X_{1}, \cdots, X_{d}, T_{1}, \cdots, T_{k}\right]$ bigraded as before. Then, $M=\bigoplus_{n \in \mathbb{N}} F_{n}(N)$ is naturally a finitely generated, bigraded $R$-module with $M^{(n)} \cong F_{n}(N)$. Now appeal to Theorem 1.

Recall that an ideal $J \subseteq I$ is said to be a reduction of $I$ if for some $n \in \mathbb{N}$ we have that $I^{n}=J I^{n-1}$. We will denote by $\rho(I)$ the minimum of $\theta(J)$ over all graded reductions $J$ of $I$. Clearly, $\operatorname{reg}(I) \geq \theta(I) \geq \rho(I)$.

Corollary 3. Let $I$ be a graded ideal of $S=k\left[x_{1}, \cdots, x_{d}\right]$ and let $P=\rho(I)$. Then there exists a constant $Q$ so that $\operatorname{reg}\left(I^{n}\right) \leq P n+Q$ for all $n \in \mathbb{N}$.

Proof. Let $J$ be a graded reduction of $I$ with $\theta(J)=P$. As above, map a bigraded polynomial ring $R$ onto $S[J t]$. Since $J$ is a reduction of $I, S[I t]$ is a finitely generated bigraded $S[J t]$-module and hence also a finitely generated bigraded $R$-module. Apply Theorem 1 to this module.

In order to improve the inequality of the corollary to an asymptotic equality, we first linearly bound $\operatorname{reg}\left(I^{n}\right)$ below by simply bounding $\theta\left(I^{n}\right)$.

Proposition 4. Let $I$ be a graded ideal of $S=k\left[x_{1}, \cdots, x_{d}\right]$ and let $P=\rho(I)$. Then $\theta\left(I^{n}\right) \geq$ Pn for all $n \in \mathbb{N}$.

Proof. Let $p \in \mathbb{N}$ be largest so that there exists $f \in I$ of degree $p$ with $f^{n} \notin \mathfrak{m} I^{n}$ for all $n \in \mathbb{N}$. Hence $I^{n}$ has a minimal generator in degree $p n$ for every $n$ and so $\theta\left(I^{n}\right) \geq p n$ for all $n \in \mathbb{N}$. It suffices to show that $p \geq P$ or equivalently that there exists a graded reduction $J$ of $I$ with $\theta(J) \leq p$.

Choose a minimal generating set $f_{1}, \cdots, f_{k}$ of $I$ of degrees $p_{1} \leq \cdots \leq p_{k}$ respectively so that $f_{j}^{n} \notin \mathfrak{m} I^{n}$ for all $n$ and $p_{i}>p_{j}=p$ for $i>j$. Set $J=\left(f_{1}, \cdots, f_{j}\right)$ and $K=\left(f_{j+1}, \cdots, f_{k}\right)$. Clearly $J$ is a graded ideal with $\theta(J)=p$ and we claim that $J$ is a reduction of $I$. This will complete the proof.

From the definition of $p$ it follows easily that, for some $n \in \mathbb{N}, K^{n} \subseteq \mathfrak{m} I^{n}$. Then $I^{n}=(J+K)^{n}=J(J+K)^{n-1}+K^{n} \subseteq J I^{n-1}+\mathfrak{m} I^{n}$. By Nakayama's lemma, it follows that $J$ is a reduction of $I$.

Theorem 5. Let I be a graded ideal of $S=k\left[x_{1}, \cdots, x_{d}\right]$ and let $P=\rho(I)$. Then there exists a constant $M \in \mathbb{N}$ so that, for all $n \in \mathbb{N}$ sufficiently large, $\operatorname{reg}\left(I^{n}\right)=$ $P n+M$.

Proof. By Proposition 4, $\operatorname{reg}\left(I^{n}\right)=P n+Q_{n}$ for non-negative integers $Q_{n}$. We will show that the sequence $Q_{n}$ is eventually constant.

Choose a graded reduction $J=\left(f_{1}, \cdots, f_{k}\right)$ of $I$ where $f_{i}$ is homogeneous of degree $p_{i}$ and $\theta(J)=P$. Let $R$ be the bigraded ring $k\left[X_{1}, \cdots, X_{d}, T_{1}, \cdots, T_{k}\right]$ mapping onto $S[J t]$ as before. Consider the Koszul complex of the bigraded $R$ module $S[I t]$ with respect to $T_{1}, \cdots, T_{k}$. All homology modules of this complex are annihilated by a power of $\left(T_{1}, \cdots, T_{k}\right)$ and hence, for all sufficiently large $n$, applying the functor $(\cdot)^{(n)}$ yields an exact complex of graded $S$-modules:

$0 \rightarrow I^{n-k}\left(-p_{1}-p_{2}-\cdots-p_{k}\right) \rightarrow \cdots \rightarrow I^{n-1}\left(-p_{1}\right) \oplus \cdots \oplus I^{n-1}\left(-p_{k}\right) \rightarrow I^{n} \rightarrow 0$. 
This complex may be used to construct a resolution of $I^{n}$ given resolutions of $I^{n-1}, \cdots, I^{n-k}$ and it follows from this construction that, for all $n$ sufficiently large, $\operatorname{reg}\left(I^{n}\right) \leq \max \left\{\operatorname{reg}\left(I^{n-1}\right)+\max _{i}\left\{p_{i}\right\}, \operatorname{reg}\left(I^{n-2}\right)+\max _{i<j}\left\{p_{i}+p_{j}\right\}-1, \cdots, \operatorname{reg}\left(I^{n-k}\right)\right.$ $\left.+p_{1}+\cdots+p_{k}-(k-1)\right\}$. Since $P=\max _{i}\left\{p_{i}\right\}, 2 P \geq \max _{i<j}\left\{p_{i}+p_{j}\right\}$ etc., this implies that $Q_{n} \leq \max \left\{Q_{n-1}, Q_{n-2}-1, \cdots, Q_{n-k}-(k-1)\right\}$.

For $n>k$, define $M_{n}=\max \left\{Q_{n-1}, \cdots, Q_{n-k}\right\}$. Then for all sufficiently large $n$, the sequence $M_{n}$ is a non-increasing sequence of non-negative integers and therefore eventually constant with value, say, $M$. The sequence $Q_{n}$ is bounded above for all large $n$ by $M$. For sufficiently large $n$, if some $Q_{n}<M$, it follows that all successive $Q$ 's are also less than $M$. But then, $M_{n}$ would also be less than $M$ for all large $n$. The contradiction shows that the sequence $Q_{n}$ is also eventually constant with value $M$.

Remarks. (1) The theorem should be compared with [BrtEinLzr, Proposition 1] and its refinements in [Brt]. Explicit determination of a $Q$ as in Corollary 3 seems to involve fairly subtle techniques. On the other hand, it may be possible to find the $M$ of Theorem 5 in the spirit of the methods of this paper.

(2) Following [SnbGot], say that a graded ideal $I$ has a linear resolution if its regularity is equal to the degree of each of its minimal generators. In an earlier version of this paper I had the following proof that Chandler's conjecture is equivalent to the statement: If $I$ has a linear resolution, so do all powers of $I$.

Proof. Clearly, Chandler's conjecture implies that statement. Conversely suppose that powers of ideals with linear resolutions also have linear resolutions. Let $I$ be an arbitrary graded ideal of $S$ with $\operatorname{reg}(I)=r$. By [SnbGot, Proposition 1.1], the graded ideal $I_{\geq r}\left(=I \cap \mathfrak{m}^{r}\right)$ has a linear resolution. Hence, so does $\left(I_{\geq r}\right)^{n}$ for all $n \in \mathbb{N}$. Since $r \geq \theta(I),\left(I_{\geq r}\right)^{n}=I^{n} \cap \mathfrak{m}^{r n}$. By [SnbGot, Proposition 1.1] again, $\operatorname{reg}\left(I^{n}\right) \leq r n=n \cdot \operatorname{reg}(I)$.

Subsequently, I was made aware of an example, attributed to Terai in [Cnc], of a monomial ideal with linear resolution whose square does not have a linear resolution in characteristic different from 2. Thus, Chandler's conjecture is false. A recent preprint of Bernd Sturmfels [Str] gives a characteristic free such example.

(3) The main result of this paper has been independently obtained in [CtkHrzTrn]. This paper also studies the regularity of the saturations of powers of an ideal.

(4) The referee has suggested that the bound

$$
\operatorname{reg}\left(I^{n}\right) \leq \max \left\{\operatorname{reg}\left(I^{n-1}\right)+\max _{i}\left\{p_{i}\right\}, \cdots, \operatorname{reg}\left(I^{n-k}\right)+p_{1}+\cdots+p_{k}-(k-1)\right\},
$$

in the proof of Theorem 5 follows easily by inductively applying the lemma: If $0 \rightarrow A \rightarrow B \rightarrow C \rightarrow 0$ is an exact sequence, then $\operatorname{reg}(C) \leq \max \{\operatorname{reg}(B), \operatorname{reg}(A)-1\}$.

\section{ACKNOWLEDGEMENTS}

I am very grateful to Irena Swanson for her preprint which kindled my interest in this question. I also thank Karen Chandler for her preprint, Dan Katz for his lectures on regularity, Radha Mohan and Shreedhar Inamdar for helpful conversations and the referee for a meticulous reading. 


\section{REFERENCES}

[BrtEinLzr] Aaron Bertram, Lawrence Ein, Robert Lazarsfeld, Vanishing theorems, a theorem of Severi, and the equations defining projective varieties, Journal of the American Mathematical Society 4 (1991), 587-602. MR 92g:14014

[Brt] Aaron Bertram, An application of a log version of the Kodaira vanishing theorem to embedded projective varieties, Preprint alg-geom/9707001.

[Chn] Karen A. Chandler, Regularity of the powers of an ideal, Communications in Algebra 25 (12) (1997), 3773-3776. CMP 98:04

[Cnc] Aldo Conca, Hilbert function and resolutions of the powers of the ideal of the rational normal curve, Preprint.

[CncHrzTrnVll] Aldo Conca, Jurgen Herzog, Ngo Viet Trung, Giuseppe Valla, Diagonal subalgebras of bigraded algebras and embeddings of blow-ups of projective spaces, American Journal of Mathematics 119 (4) (1997), 859-902. CMP 97:17

[CtkHrzTrn] Dale Cutkosky, Jurgen Herzog, Ngo Viet Trung, Asymptotic behaviour of the Catelnuovo-Mumford regularity, Preprint.

[Snb] David Eisenbud, Commutative Algebra with a View Toward Algebraic Geometry, Springer-Verlag, 1995. MR 97a:13001

[SnbGot] David Eisenbud, Shiro Goto, Linear free resolutions and minimal multiplicity, Journal of Algebra 88 (1984), 89-133. MR 85f:13023

[GrmGmgPtt] A. V. Geramita, A. Gimigliano, Y. Pitteloud, Graded Betti numbers of some embedded rational $n$-folds, Mathematische Annalen 301 (1995), 363-380. MR 96f: 13022

[HoaTrn] Le Tuan Hoa, Ngo Viet Trung, On the Castelnuovo-Mumford regularity and the arithmetic degree of monomial ideals, Mathematische Zeitschrift, To appear.

[Res] D. Rees, Reduction of modules, Mathematical Proceedings of the Cambridge Philosophical Society 101 (1987), 431-448. MR 88a:13001

[SmtSwn] Karen E. Smith, Irena Swanson, Linear bounds on growth of associated primes, Communications in Algebra, 25 (1997), 3071-3079. CMP 97:17

[Str] Bernd Sturmfels, Four counterexamples in combinatorial algebraic geometry, Preprint.

[Swn] Irena Swanson, Powers of ideals: Primary decompositions, Artin-Rees lemma and regularity, Mathematische Annalen 307 (1997), 299-313. MR 97j:13005

The Institute of Mathematical Sciences, Chennai, India 600113

E-mail address: vijay@imsc.ernet.in 